\title{
Integration of In Silico and In Vitro Approach to Reveal the Anticancer Efficacy of Virgin Coconut Oil
}

\author{
Babita Pruseth $^{1}$, Silvi Banerjee ${ }^{1}$, Amit Ghosh ${ }^{1^{*}}$
}

\begin{abstract}
Virgin coconut oil (VCO) has antioxidant properties and is being increasingly used as nutraceuticals and cosmeceuticals. It also has a long history of ethnopharmacological use. Anticancer effect of VCO has been reported in several articles. The main bottleneck of exploring the anticancer efficacy of VCO is the difficulty in identification and validation of target proteins and their regulated pathways. The work plan was in-silico analysis using Comparative Toxicogenomics Database (CTD) and STRING. CTD curated and integrated data for more than 5700 gene-disease and 2000 chemical-disease relationship. Medium Chain Fatty Acids (MCFAs) from VCO like Lauric acid, Caprylic Acid, Capric Acid, and Myristic acid can target almost 17 cancer-associated proteins. An attempt was made to identify the target proteins and their pathways regulated by VCO. We analyze curated and inferred VCO-gene expression data and illustrate the impact of VCO exposure on cancer-related gene network and molecular function. In enriched pathway analysis, it has been evident that all of them are the part of different cancer-associated pathways (Neoplasms, Digestive System Neoplasms, Urogenital Neoplasms, Liver Neoplasms). This response may mimic the biological response to VCO. In silico result was tested by in vitro study and VCO kill the Human hepatocellular carcinoma cell lines (hepG2). Based on the findings of this study and several published studies it is proposed that a VCO may have immense potential as a botanical product against cancer.
\end{abstract}

Key words: Virgin Coconut Oil, Liver, and Oral cancer, Protein targets, Pathway analysis, Human Intervention trial

\section{Introduction}

Anticancer effect of Virgin Coconut Oil (VCO) has been reported in several articles. A large number of the published article indicates the anticancer potential of VCO, especially in the colon, breast, lung, Liver and oral cavity. Lim-Sylianco (1987) published a 50-year literature review showing the anticancer effect of coconut oil (1). Cohen et al. (1986) showed that coconut oil was far more protective than unsaturated oil in chemically induced colon and breast cancer. In his study, 32\% corn oil eater developed colon cancer, as compared to only 3\% of coconut oil eater (2). Recently Law et al. (2014) reported that VCO consumption during chemotherapy helped improve functional status and global quality of life of breast cancer patients. It also reduces the symptom related to the side effect of chemotherapy (3). MCFA compositions are altered in breast cancer tissue (4). The lauric acid is a major component of the VCO and ranged from 46.36 - $48.42 \%$. Lauric acid-induced apoptosis in colon cancer cell by triggering oxidative stress (5). Moreover, the anticancer activity of VCO against breast cancer cell line (SkBr-3) reported by Calderon et al. (6). Yahaya et al. also reported the anticancer effect of VCO on lung cancer cells. VCO induced apoptosis in NCI-H1299 and A549 cell line (7). Recently Enos et al. 2016 reported that the protective role of coconut oil in colon cancer induced by azoxymethane (AOM)/dextran sulfate sodium (DSS) in a murine model (8). Famurewa et al. 2017 reported the antioxidant and hepatoprotective effects of VCO supplementation against hepatotoxicity and oxidative damage induced by anticancer drug methotrexate (MTX) in rats (9).

Despite the diverse and serious beneficial effect of VCO, its molecular mechanism of action yet to be identified. To translate, the most relevant missing data are to identify the target protein and pathways, followed by human intervention trials. To identify target protein and pathway associated with the anticancer effect of VCO, Comparative Toxicogenomics Database (CTD) and STRING was used. In

\footnotetext{
${ }^{1}$ Department of Physiology, All India Institute of Medical Sciences (AIIMS), Bhubaneswar, India

${ }^{*}$ Corresponding Author
} 
addition to that anticancer efficacy of VCO and Fractionate coconut oil was tested in liver cancer cell line (HepG2).

\section{Method}

\section{Curation process (VCO-gene-disease interaction)}

CTD provides the information about VCOgene-Disease relationship by integrating data curated from the scientific literature. Candidate references for curation in CTD were collected by querying PubMed for co-occurrence of associated disease and chemical name. The fatty acid composition of VCO range from C8-C18 and predominant MCFAs are Lauric Acid, Caprylic Acid, Capric Acid, and Myristic Acid. The fatty acid name was used as a query term to retrieve the top 10 associated diseases. Each compound associated disease and corresponding gene expression signature was evaluated against a pre-existing literature to predict they're possible anticancer efficacy. The analysis reported here was based on data downloaded and analyzed in July 2018.

Compound Signature Matching: All chemical-disease links known as a marker or therapeutic agent in CTD. MCFAs of VCO induced gene expression signatures were evaluated against pre-existing disease-associated gene signature library in order to make a prediction of their anticancer efficacy. Compounds linked to most relevant disease were explored using CTD. However, in order to reduce the noise and to focus on the most relevant information, only the top 5 associated diseases with each compound were considered for further study. Among these only chemical - cancer data with CTD interference score above 5 were considered for the chemical-disease association.

Disease analysis: Curated and inferred interaction VCO MCFAs-gene were combined. Total 17 genes, selected by compound-diseasegene interaction were considered for further study. Among these genes, a number of genes overlapped with major VCO compounds. Total 40 genes interact with MCFAs of VCO. These data indicate that major VCO compounds share some common molecular activity, although the overall biological effect or the molecular network they invoke may be compound specific. This approach may reasonably mimic a biological response to VCO. CTD set analyzer tool was used to demonstrate the combined gene network as well as disease and pathway enrichment.
Gene Ontology enrichment analysis: The GO is an independent annotation resource used by bio curators to characterize gene product's molecular function (GO-MF), the Cellular compartment (GO-CC), and Biological process (GO-BP). The analysis was derived from data available in CTD in July 2018. GO-CC query term included "plasma membrane protein", "nucleus" and "intracellular compartment" etc.

Protein-protein interaction: Predicted protein-protein interactions (PPI) were obtained from the Search Tool for the Retrieval of Interacting Genes/Proteins (STRING) database. PPI network is a useful tool to decipher the mechanism of action. In PPI, nodes represent protein and edges represent interaction ie. the binding of one protein to another. These proteins were analyzed by STRING database to create interacting proteins network.

Co-expression: Co-expression analysis relies on the hypothesis that highly correlated gene are biologically related. Co-expression network analysis utilizes entire measured transcription to help to determine gene function and mode of action.

Cell Culture: This study is a cell culturebased study. The study was conducted by using HepG2 (hepatocyte carcinoma cell line) cell line. Cells were obtained from the National Center for Cell Science, Pune (NCCS), India. All the cell cultures were performed in a class two laminar flow cabinet and the cells were maintained at $37^{\circ} \mathrm{C}$ in a humidified atmosphere of 5\% CO2 and 95\% air under aseptic condition. Chemicals were purchased from different known companies. A standard protocol was followed for the abovementioned assay.

Treatment of Cells: Cell line was treated with different concentrations of Virgin Coconut Oil (VCO), and Fractionated Coconut Oil (FCO). Cells were harvested after $72 \mathrm{hr}$ of incubation.

Determination of Cytotoxicity and Cell Viability by MTT Assay: Cells were seeded into $35 \mathrm{~mm}$ culture dishes containing suitable growth media and were maintained at $37^{\circ} \mathrm{C}$ in a humidified atmosphere of 5\% CO2 and 95\% air under aseptic condition. After 24 hours, cells were treated with different concentrations of VCO and Fractionated coconut oil (FCO). FCO contain C8:0-54.15\%; C10:0-45.11\%. Dose-response was verified using 3-(4,5-Dimethylthiazol-2- 
Figure 1. Showing the top 10 diseases associated with SCFAs of VCO (X axis represents the types of diseases associated with each fatty acids)
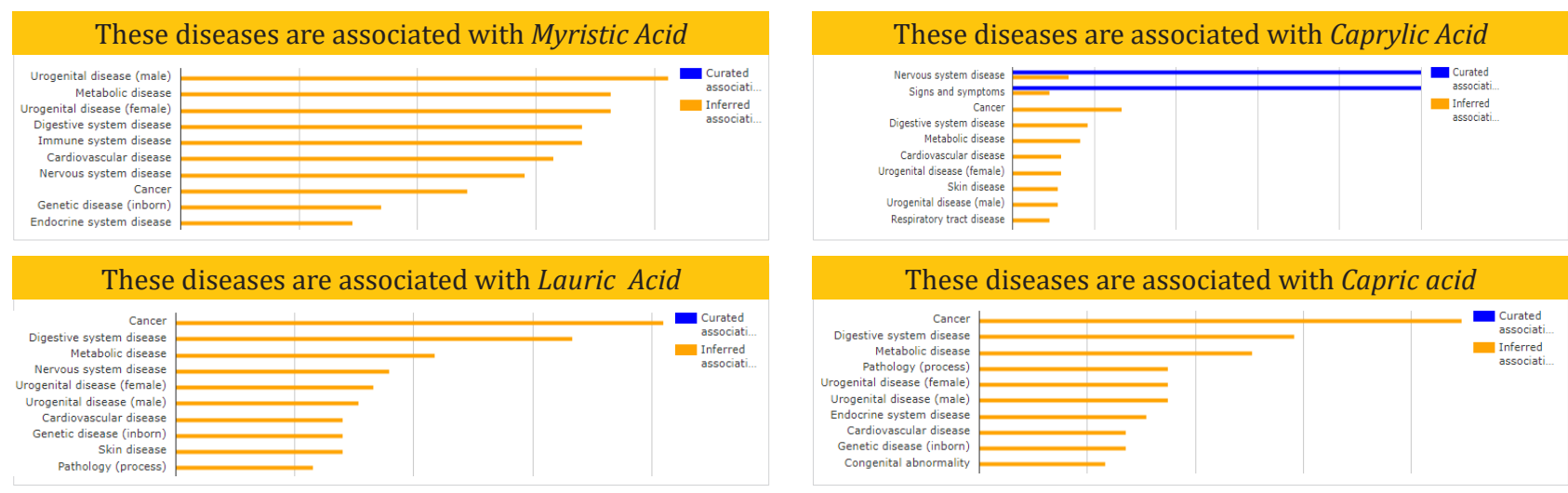

yl)-2,5-diphenyltetrazolium bromide (MTT) analysis. Each treatment was repeated 3 times. The cells were then incubated with MTT in the growth medium for $4 \mathrm{~h}$ at $37^{\circ} \mathrm{C}$. Cell viability was evaluated by comparison with a control culture (assumed to be $100 \%$ viable). VCO and FCO dose and the incubation period were standardized on the basis of a previous study.

Statistical Analysis: The whole procedure was repeated three times. The results were presented as mean \pm Standard Error of the Mean (SEM). Significant changes were assessed by Student's t-test. A value of $\mathrm{P}<0.05$ was considered significant.

\section{Result}

To determine whether VCO affect cancerassociated gene, we explore cancer-associated gene set interacting with different MCFAs of VCO. The major SCFAs of VCO is Lauric acid, Caprylic acid, Capric Acid, and Myristic acid predominantly associated with the cancerassociated pathway (Fig. 1). The top 5 associated diseases with each compound of VCO were selected based on their inference score, which shows the degree of similarity between CTD chemical-gene-disease networks and a similar scale-free random network and subjected to further study.

Four components interact with 17 cancerassociated genes (ALB, ATF3, CAV1, CLDN1, CXCL8. CYP2E1, CYP4B1, MAPK14, NR1H2, POR, PPARA, PPARG, PPARGC1A, RELA, TTR, UGT2B7) which are associated with a neoplasm (digestive system, liver, lung, urogenital etc.). The disease was subdivided into a specific type of malignancies. (Table 1).

Pathway analysis showed that the network was enriched with the role in Neoplasms $(a l b$, atf3, cav1, cldn1, cxcl8, cyp2c9, cyp2e1, cyp4b, mapk14, nr1h2, por, ppara, pparg, ppargc1a, rela, ttr, ugt2b7), Digestive System Neoplasms (alb, atf3, cav1, cxcl8, cyp2e1, mapk14, nr1h2, ppara, pparg, rela), Urogenital Neoplasms (atf3, cav1, cxcl8, cyp2e1, cyp4b1, por, ppara, rela, ugt2b7), Liver Neoplasms (atf3, cxcl8, cyp2e1, mapk14, nr1h2, ppara, pparg).

GO pathway analysis shows most of gene product location is intracellular and gene products are the transcription factor and have DNA, RNA, and protein binding activity (Table 2 ).

The anticancer implication of VCO- genes network is consistent with KEGG pathway and Disease association. CTD based analysis provide candidate gene that may help to explain the underlying mechanism of action of VCO.

We illustrate the PPI network of the candidate genes, which revealed the confirmed interaction of the following protein proved by experiment and from a curated database. Besides this inferred protein-protein interaction also revealed based on protein homology, gene neighbourhood, text mining, and protein homology. Most of this protein co-expressed in human and other species. Pathway analysis from protein-protein interaction (STRING) also reveals that cancer-related pathways are associated with candidate genes (Fig 2).

As mentioned above we have investigated the effect of VCO by adding the DMSO as a solvent in 1:5 ratio. The vehicle control (DMEM and DMSO in 5:1 ratio) was also used. In the case of 


\begin{tabular}{|c|c|c|}
\hline $\begin{array}{l}\text { Disease Name } \\
\text { (Top } 10 \text { out of } 27)\end{array}$ & $\begin{array}{c}\text { Annotated } \\
\text { Genes } \\
\text { Quantity } \\
\end{array}$ & Annotated Genes \\
\hline Neoplasms & 16 & $\begin{array}{l}\text { ALB|ATF3|CAV1|CLDN1|CXCL8|CYP2E1|CYP4B1|MAPK14|NR1H2|POR| } \\
\text { PPARA|PPARG|PPARGC1A|RELA|TTR|UGT2B7 }\end{array}$ \\
\hline Neoplasms by Site & 15 & $\begin{array}{l}\text { ALB|ATF3|CAV1|CLDN1|CXCL8|CYP2E1|CYP4B1|MAPK14|NR1H2|POR| } \\
\text { PPARA|PPARG|RELA|TTR|UGT2B7 }\end{array}$ \\
\hline $\begin{array}{l}\text { Digestive System } \\
\text { Neoplasms }\end{array}$ & 10 & ALB|ATF3|CAV1|CXCL8|CYP2E1|MAPK14|NR1H2|PPARA|PPARG|RELA \\
\hline $\begin{array}{l}\text { Urogenital } \\
\text { Neoplasms }\end{array}$ & 9 & ATF3|CAV1|CXCL8|CYP2E1|CYP4B1|POR|PPARA|RELA|UGT2B7 \\
\hline Liver Neoplasms & 7 & ATF3|CXCL8|CYP2E1|MAPK14|NR1H2|PPARA|PPARG \\
\hline $\begin{array}{l}\text { Neoplasms by } \\
\text { Histologic Type }\end{array}$ & 10 & $\begin{array}{l}\text { ALB|CXCL8|CYP2E1|MAPK14|NR1H2|POR|PPARA|PPARG|PPARGC1A| } \\
\text { RELA }\end{array}$ \\
\hline $\begin{array}{l}\text { Neoplasms, } \\
\text { Glandular and } \\
\text { Epithelial }\end{array}$ & 9 & ALB|CXCL8|CYP2E1|NR1H2|POR|PPARA|PPARG|PPARGC1A|RELA \\
\hline Lung Neoplasms & 7 & ALB|ATF3|CAV1|CXCL8|CYP2E1|MAPK14|TTR \\
\hline $\begin{array}{l}\text { Respiratory Tract } \\
\text { Neoplasms }\end{array}$ & 7 & ALB|ATF3|CAV1|CXCL8|CYP2E1|MAPK14|TTR \\
\hline $\begin{array}{l}\text { Thoracic } \\
\text { Neoplasms }\end{array}$ & 7 & ALB|ATF3|CAV1|CXCL8|CYP2E1|MAPK14|TTR \\
\hline
\end{tabular}

Table 1. cancer-associated genes associated with different cancer (CTD)

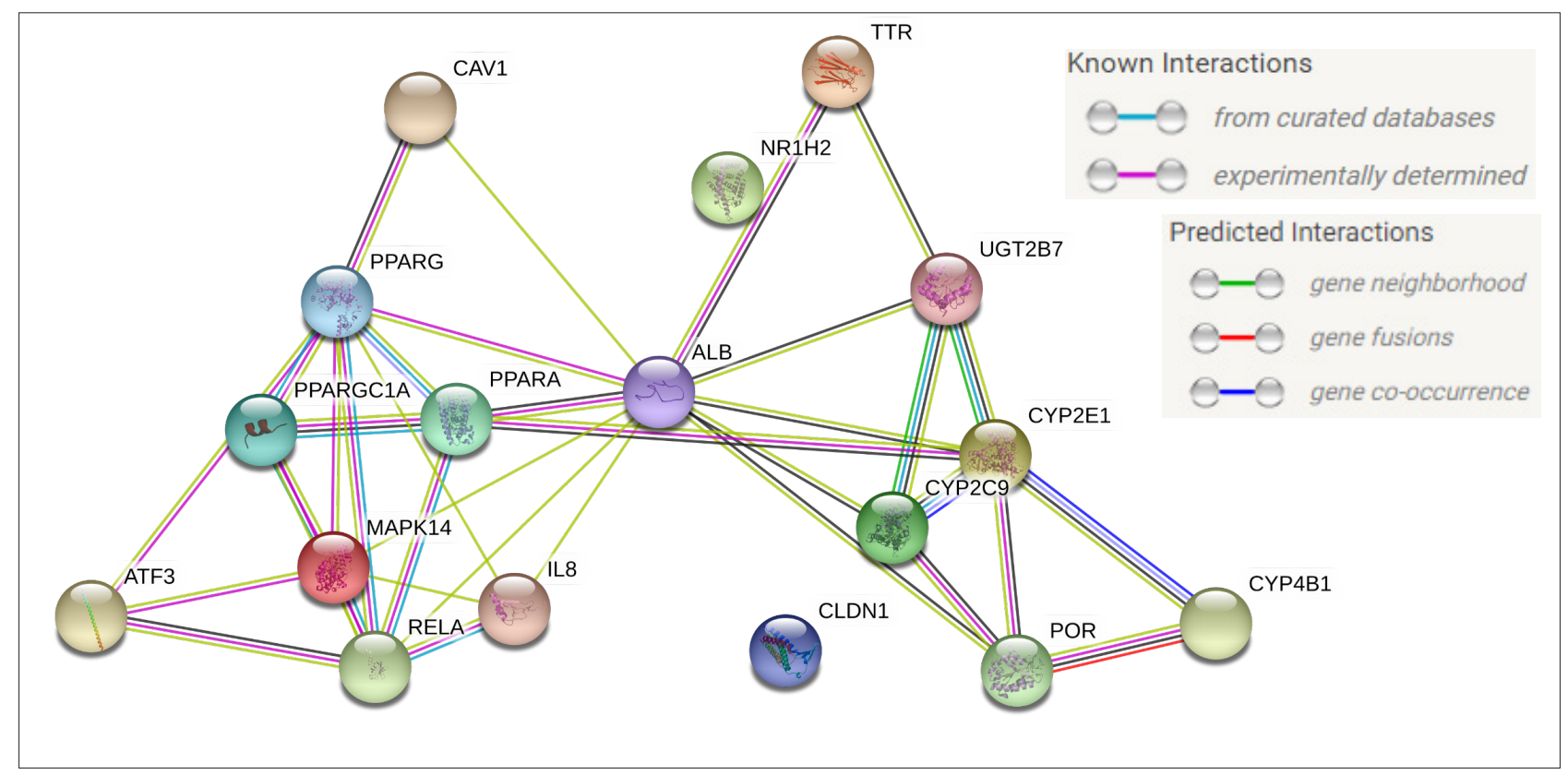

Figure 2. Interaction of protein which expression is modulated by VCO 


\begin{tabular}{lr}
\hline GO Molecular Function (top 10 out of 21) & Gene \\
\hline enzyme binding & 9 \\
organic cyclic compound binding & 12 \\
transcription factor binding & 6 \\
heterocyclic compound binding & 11 \\
oxygen binding & 3 \\
RNA polymerase II proximal promoter & 5 \\
sequence-specific & \\
DNA binding & 14 \\
protein binding & 5 \\
proximal promoter sequence-specific DNA & 3 \\
binding & \\
nuclear receptor activity & 3 \\
transcription factor activity, direct ligand- & \\
regulated sequence-specific DNA binding & \\
\hline G0 Biological Process (top 10 out of 218) & Gene \\
\hline monocarboxylic acid metabolic process & 9 \\
fatty acid metabolic process & 8 \\
response to drug & 10 \\
cellular response to chemical stimulus & 13 \\
response to chemical & 14 \\
small molecule metabolic process & 11 \\
response to lipid & 9 \\
lipid metabolic process & 10 \\
carboxylic acid metabolic process & 9 \\
cellular response to lipid & 8 \\
\hline
\end{tabular}

\begin{tabular}{lr}
\hline GO Cellular compartment (top 10 out of 218) & Gene \\
\hline intracellular & 16 \\
intracellular organelle part & 14 \\
organelle part & 14 \\
intracellular membrane-bounded organelle & 14 \\
cell part & 16 \\
cell & 16 \\
membrane-bounded organelle & 14 \\
intracellular part & 15 \\
intracellular organelle & 14 \\
\hline KEGG Pathway (top 10 out of 218) & Gene \\
\hline Hepatitis C & 5 \\
Insulin resistance & 4 \\
Non-alcoholic fatty liver disease (NAFLD) & 4 \\
Shigellosis & 3 \\
Epithelial cell signaling in Helicobacter pylori & 3 \\
infection & \\
Adipocytokine signaling pathway & 3 \\
RIG-I-like receptor signaling pathway & 3 \\
Pertussis & 3 \\
Salmonella infection & 3 \\
Longevity regulating pathway & 16 \\
\hline Enriched diseases (top 10 out of 111) & Gene \\
\hline Neoplasms & 16 \\
Neoplasms by Site & 15 \\
Female Urogenital Diseases & 12 \\
Cemale Urogenital Diseases and Pregnancy & 12 \\
Male Urogenital Diseases & 11 \\
Endocrine System Diseases & \\
Nutritional and Metabolic Diseases & \\
Digestive System Neoplasms & \\
Liver Diseases & \\
Diabetes Mellitus, Experimental & 10 \\
\hline
\end{tabular}

Table 2. GO and KEGG annotation for VCO SCFAs interacting gene 


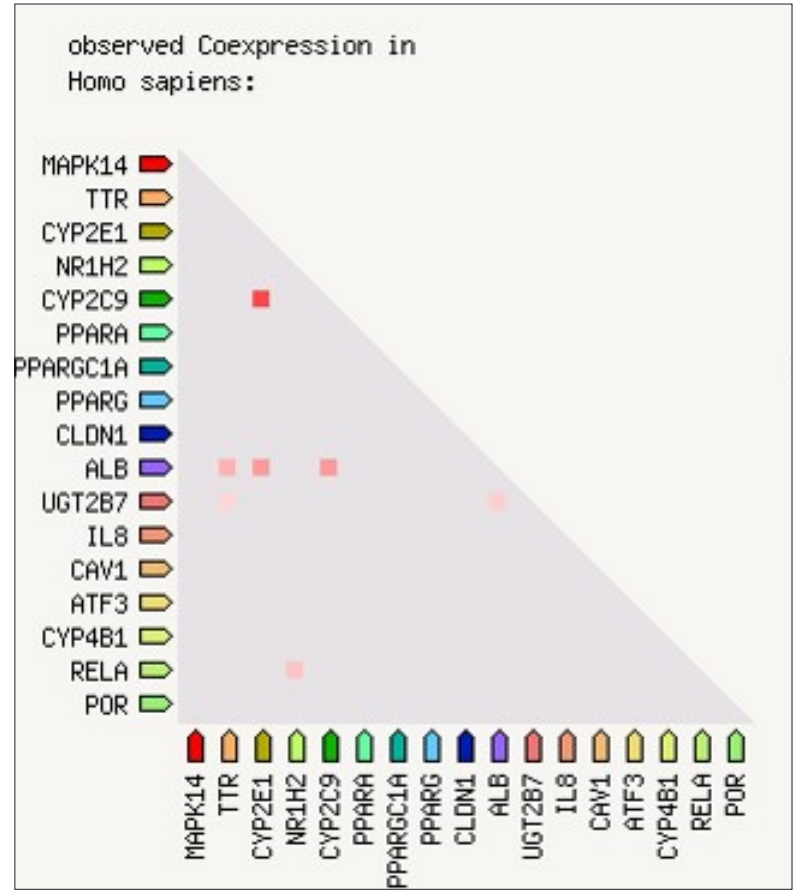

Figure 3. Showing the co-expression of VCO cancer-associated genes

HepG2 cells, the VCO along with solvent showing the significant cell death with $20 \%, 40 \%, 60 \%$, and $80 \%$ when compared to control (Fig. 4). We observed the similar results when the same cell lines were treated with Fractionated coconut oil (FCO) along with solvent (Fig. 5). All experiments were repeated three times.
Phase contrast images of control and treated with $20 \%$ of FCO or VCO with a solvent in the HepG2 cell lines are given below (Fig. 4 and 5).

The result of the in-vitro cell culturebased study clearly indicates that VCO may have some anticancer component which is also present in FCO.

The fatty acid may be the MCFAs. As in all VCO, amount of Lauric acid range from 46\%$48 \%$, so we have interested about in-silico analysis of the possible anticancer role of Lauric acid along with other fatty acids in VCO.

\section{Discussion}

To determine whether different fatty acid of VCO regulate a cancer-associated gene, we explore the interacting gene set with neoplasm associated gene set. VCO-disease relationship is clearly labelled as either the direct or inferred. A direct relationship is explored by integration of gene-disease relationship from the OMIM database. The inferred relationship is established by integrating curated genedisease data. It was also found that SCFAs of VCO can target almost 17 cancer-associated proteins. Almost $50 \%$ of VCO is Lauric Acid which interacts with 18 genes and associated with several diseases among which cancer, digestive disease, Metabolic Disease, Nervous System Disease, and Urogenital Diseases are top five. Caprylic Acid interacts with 14 genes and associated with several diseases- Nervous System Disease, Sign and Symptoms, cancer,

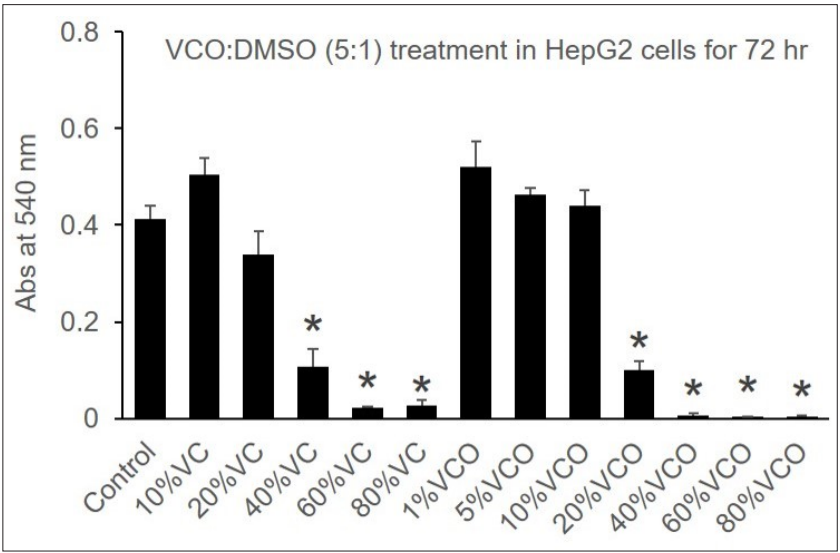

Figure 4. Cell viability assay after treatment with various concentrations of VCO and DMSO (in 5:1 ratio) in HepG2 cells for $72 \mathrm{hr}$. Student's-t test was performed to analyze the significant difference between the control and VCO treated cells. Mean \pm SEM. ${ }^{*} \mathrm{p} \leq 0.05$

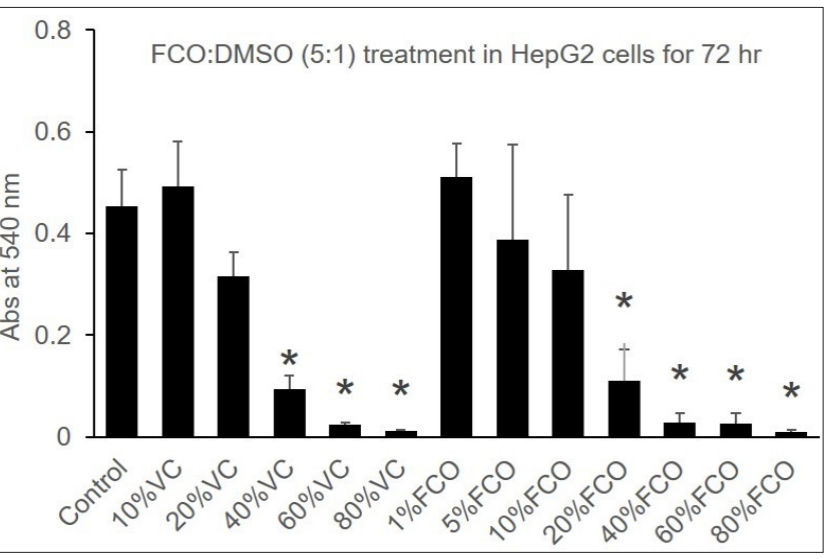

Figure 5. Cell viability assay after treatment with various concentrations of FCO and DMSO (in 5:1 ratio) in HepG2 cells for $72 \mathrm{hr}$. Student's-t test was performed to analyze the significant difference between the control and VCO treated cells. Mean \pm SEM. ${ }^{*} \mathrm{p} \leq 0.05$ 


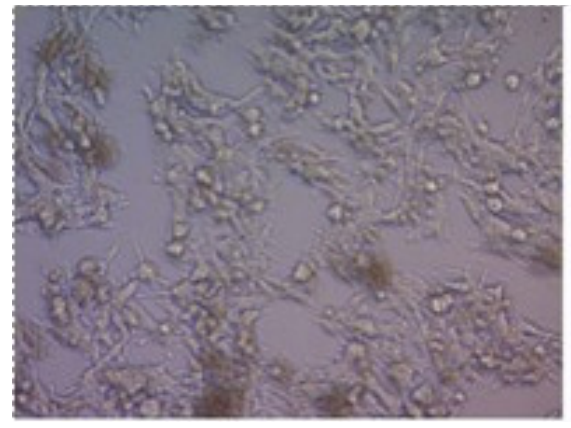

Control-HepG2 cells

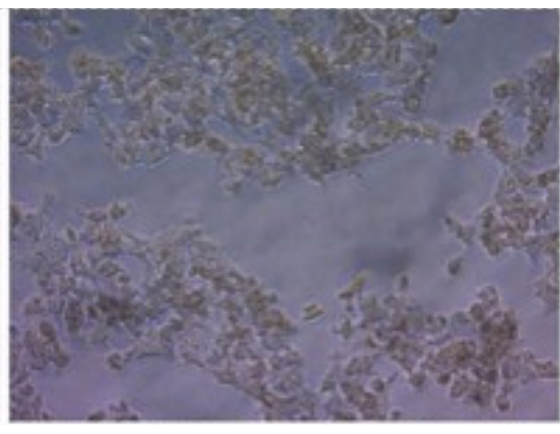

20\% VCO treatment in HepG2 cells

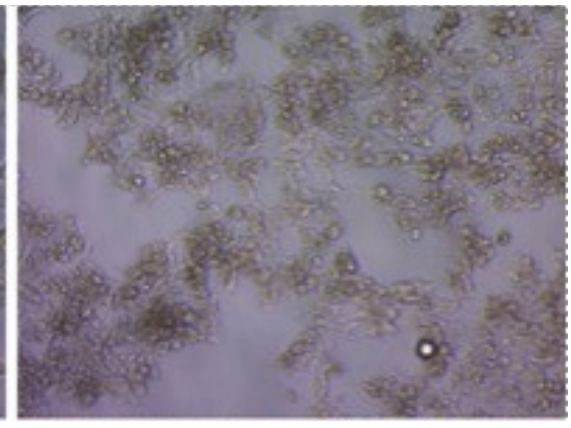

20\% FCO treatment in HepG2 cells

Figure 6. Phase contrast images (20x) of HepG2 and KB cells after treatment with 20\% VCO: DMSO (1:5) and FCO: DMSO (1:5)

digestive disease, and Metabolic Disease are top five. Capric acid also interacts with 8 genes and top five associated diseases are Cancer, Digestive disease, Metabolic Disease, Nervous System Disease, and Urogenital Diseases. Remarkably top five associated disease of 3 MCFAs of VCO are same. In case of Lauric acid 10 out of 18 genes, Caprylic acid 8 out of 14 genes and Capric acid 6 out of 8 genes associated with Cancer.

CTD analysis indicated that almost all of 17 proteins identified are the part of the cancer pathway and apoptosis. VCO regulated the expression of several genes. The top 10 Genes has been associated with selected pathway and disease association, indicating VCO may have modulate disease pathology of cancer (Table 1). Kyoto Encyclopaedia of Genes and Genomes (KEGG) pathway enrichment analysis shows to 10 pathways shows their associated with cancer (Table 2). KEGG is a database resource for understanding high-level functions and utilities of the biological system, such as the cell, the organism and the ecosystem, from genomic and molecular-level information. These target genes were involved with many pathways and related disease like, Neoplasms, Urogenital Diseases, Digestive System Neoplasms, metabolic Disorder etc. The Gene Ontology (GO) analysis study was predicted the top 10 associated biological processes, cellular components and molecular functions related to the given set of targets gene that are regulated by VCO. The GO describes the biological domain with respect to three aspects: Molecular Function, biological process and cellular components. In enriched pathway, KEGG analysis came up with the diagram of cancerassociated pathways and all of them are the part of different cancer-associated pathways. The list of the VCO target genes (proteins) was subjected to STRING (Version: 11.0) analysis to reveal functional interactions between the VCO target proteins (Fig 2). Each node represents a protein, and each edge represents an interaction. STRING analysis was used to visualize the relationship amongst VCO target genes and Fig 3 shows the co-expression of target genes.

Reportedly MCFAs of VCO can be targeted to the liver and oral cavity. So to test the in silcio results, the effect of VCO was tested in on HepG2 liver cancer cells. Cell viability assay was done and a statistically significant minimum dose of $20 \%$ VCO was found to be effective against HepG2 liver cancer cells and 40\% VCO almost kill the entire liver cell. As per human protein atlas out of 17 genes, the alb, ttr, rela, ppara, mapk14, and por RNA expression are significantly high in HepG 2 cell followed by medium level RNA expression of ugt2b7, cyp2e1, ppargc1a, atf3, pparg, nr1h2, and cldn1. As per in silico result, above mention gene are associated with liver neoplasm. In cell culture experiment, the effect of VCO on HepG2 cell also support the anticancer efficacy of VCO. In this experimental and in-silico analysis along with previously published result indicate VCO may have potent anticancer efficacy. However, the most relevant data that would support a protective effect of VCO on the treatment of Liver cancer are human intervention trials. A multi-centric, cost-effective trial may be initiated to test the therapeutic efficacy of VCO against liver neoplasm.

Today, drug discovery against cancer is one of the goals frequently pursued around the world. Natural products from plants are the endless source of new pharmaceuticals. Till now, three botanical drugs have been approved by FDA. Sinecatechins (VEREGEN ${ }^{\circledR}$ ) (15) was the first botanical drug approved in 2006. The second drug is Crofelemer (FULYZAQ $\left.^{\mathrm{TM}}\right)^{(16)}$, approved by FDA in 2012 and the third drug is GRASTEK ${ }^{\circledR}{ }^{(17)}$, approved 
in 2017. Botanical drugs have quick and large market acceptance and are quite a financial success as well. To upgrade VCO from potential anti-cancer agent to the status of 'Botanical Drug' human intervention trial is essential. A quick review of the published papers indicates that until now no human intervention trial was conducted with VCO as a preventive drug against liver cancer. Use of VCO for therapeutic purpose as a drug will enhance its value and economically benefit coconut industry.

Drawback: The interesting in silico result is supported by cell culture-based study. Though the outcome is very interesting, but it is a preliminary result. More detail study and clinical trial is required to establish the anticancer potential of VCO.

Conclusion: The outcome of this preliminary study indicates the anticancer potential of VCO.

Several studies indicate the anticancer effect of Virgin coconut oil (VCO) especially in the colon, breast, lung, Liver and oral cavity. Despite the diverse and serious beneficial effect of VCO, its molecular mechanism of action yet to be identified. The present study was attempt to identify the target proteins and their pathways regulated by VCO through in silico approach using Comparative Toxicogenomic Database (CTD) and STRING and again the in silico result was examined by in vitro study to test the anticancer efficacy of VCO on liver cancer cell line (HepG2). CTD set analyzer tool was used to demonstrate the combined gene network as well as disease and functional enrichment. The fatty acid composition of VCO range from C8-C18 and predominant MCFAs are Lauric Acid, Caprylic Acid, Capric Acid, and Myristic Acid were exposed to CTD to identify VCO-gene-Disease relationship, from where the top 5 associated diseases with each compound of VCO were picked out by the chemical-disease interaction and a number of 17 candidate genes were selected by the compound-disease-gene interaction. Pathway enrichment analysis indicated that almost all of 17 proteins identified are the part of the cancer pathway and apoptosis, where the GO analysis showed that most of gene product location is intracellular and gene products are the transcription factor and have DNA, RNA, and protein binding activity and STRING analysis revealed the functional interactions between the VCO target proteins. Further the in-silico result was extended to cell culture experiment to test the effect of VCO on HepG2 liver cancer cells, where VCO was found to be effective against HepG2 liver cancer cells and kill the entire liver cell. As per human protein atlas out of 17 genes, the alb, ttr, rela, ppara, mapk14, and por RNA expression are significantly high in HepG 2 cell followed by medium level RNA expression of ugt2b7, cyp2e1, ppargc1a, atf3, pparg, nr1h2, and cldn1. Hence it was proved the association of the 17 genes with liver neoplasm and from the cell culture study, the effect of VCO on HepG2 cell also support the anticancer efficacy of VCO. Thus, the present study along with previously published result indicate VCO may have potent anticancer efficacy, which could serve as useful sources for new anticancer agents for the treatment of Liver cancer.

\section{Conflict of Interest}

There are no conflicts of interest.

\section{Acknowledgment}

This work has been supported by AIIMS Bhubaneswar.

\section{References}

Lim Sylianco C. Y. 1987. Anti-carcinogenic effects of coconut oil. Philips J. Coconut Studies, 2(12): 89-102.

Cohen L. A., Thompson D. O., Maeura Y., Choi K., Blank M. E., Rose D. P. 1986, Jul. Dietary fat and mammary cancer. I. Promoting effects of different dietary fats on N-nitrosomethylurea-induced rat mammary tumorigenesis. J Natl Cancer Inst., 77(1): 33-42.

Law, K. S., Azman, N., Omar, E. A., Musa, M. Y, Yusoff, N. M, Sulaiman, S. A. \& Hussain, N. H. N. 2014. The Effects of Virgin Coconut Oil (VCO) as Supplementation on Quality of Life (QOL) among Breast Cancer Patients. Lipids in Health and Disease, 13, 139.

Conceição L. L., Dias M. M., Pessoa M. C., Pena G. D., Mendes M. C., Neves C. V., Hermsdorff H. H., Freitas R. N., Peluzio M. D. 2016, Nov 29. Difference in fatty acids composition of breast adipose tissue in women with breast cancer and benign breast disease. Nutr Hosp., 33(6): 1354-1360. 
Fauser J. K., Matthews G. M., Cummins A. G., Howarth G. S. 2013. Induction of apoptosis by the medium-chain length fatty acid lauric acid in colon cancer cells due to induction of oxidative stress. Chemotherapy, 59(3): 214-24.

Calderon J., Brillantes J., Buenafe M., Cabrera N., Campos E., Canoy I., Capili C., Carasco M., Cielo P., Co M., Collantes P., Concepcion F., Concha J., de la Cruz R., de Vera A., de Vera R., Chung F., Ji meno C., Valencia C. 2009. Virgin Coconut Oil Inhibits skbr-3 breast cancer cell proliferation and synergistically enhances the growth inhibitory effects of trastuzumab (herceptin ${ }^{\mathrm{TM}}$ ). Eur J Med Res, 14(Supplement II): I-XXII, 1-208

Yahaya, Badrul \& Sulaiman, Siti Amrah \& Yusop, Rahimi. 2015. Apoptosis in lung cancer cells induced by virgin coconut oil. Regenerative Research, 4: 1-7.

Enos R. T., Velázquez K. T., McClellan J. L., Cranford T. L., Nagarkatti M., Nagarkatti P. S., Davis J. M., Murphy E. A. 2016, Jun 1. High-fat diets rich in saturated fat protect against azoxymethane/dextran sulfate sodium-induced colon cancer. $A m \mathrm{~J}$ Physiol Gastrointest Liver Physiol, 310(11): G906-19.

Famurewa A. C., Ufebe O. G., Egedigwe C. A., Nwankwo O. E., Obaje G. S. 2017, Mar. Virgin coconut oil supplementation attenuates acute chemotherapy hepatotoxicity induced by anticancer drug methotrexate via inhibition of oxidative stress in rats. Biomed Pharmacother, 87: 437-442.
Rudin et al.. 2003, Dec 15. An Attenuated Adenovirus, ONYX-015, as Mouthwash Therapy for Premalignant Oral Dysplasia. J Clin Oncol, 21(24): 4546-4552.

Mattingly C. J., Colby G. T., Forrest J. N., Boyer J. L. 2003, May. The Comparative Toxicogenomics Database (CTD). Environ Health Perspect, 111(6): 793-5.

Szklarczyk, Damian et al. 2018, Jul 15. The STRING Database in 2017: QualityControlled Protein-protein Association Networks, Made Broadly Accessible. Nucleic Acids Research, 45, Database issue (2017): D362-D368. PMC. Web.

Li Y. H., Yu C. Y., Li X. X., Zhang P., Tang J., Yang Q., Fu T., Zhang X., Cui X., Tu G., Zhang Y., Li S., Yang F., Sun Q., Qin C., Zeng X., Chen Z., Chen Y. Z., Zhu F. 2018, Jan 4. Therapeutic target database update 2018: enriched resource for facilitating bench-to-clinic research of targeted therapeutics. Nucleic Acids Res, 46(D1): D1121-D1127.

Kanehisa M. 2002. The KEGG database. Novartis Found Symp., 247: 91-101, discussion 1013, 119-28, 244-52. 